ARQGA / 1063

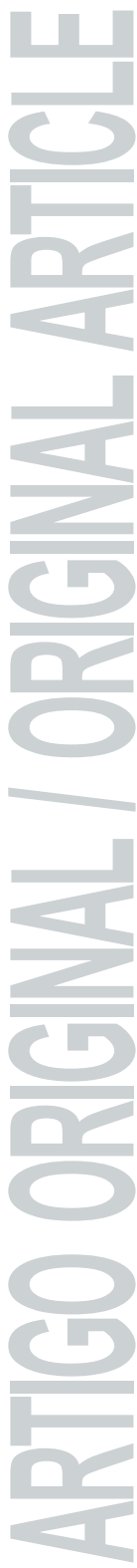

\title{
ESTADO NUTRICIONAL E AVALIAÇÃO DIETÉTICA DE PACIENTES GASTRECTOMIZADOS
}

\author{
Mayra Mayumi KAMIJI e Ricardo Brandt de OLIVEIRA
}

RESUMO - Racional - O aspecto nutricional é crucial na cirurgia de gastrectomia e a reconstrução mais adequada do canal alimentar deve ser considerada para se reduzir o risco de desnutrição. A causa da desnutrição pós-gastrectomia não é bem determinada, mas os mecanismos que levam à desnutrição são evidentemente multifatoriais. Objetivo - Avaliar o estado nutricional de pacientes submetidos a diferentes tipos de reconstrução cirúrgica após gastrectomia total ou subtotal. Pacientes e Métodos - Foram avaliados 50 pacientes submetidos a gastrectomia 0,5-39 anos antes, 7 com reconstrução a Billroth I, 26 a Billroth II, 3 a Henley e 14 a Y-de-Roux, e indicação cirúrgica de câncer em 21 dos pacientes. Foram utilizadas avaliação nutricional subjetiva e antropométrica, e avaliação dietética pelo recordatório alimentar. Resultados - Segundo a avaliação nutricional subjetiva, 6 dos 50 pacientes eram desnutridos moderados. O índice de massa corporal médio dos pacientes foi de 22 $\pm 4,75 \mathrm{~kg} / \mathrm{m}^{2}$, a ingestão média diária de $1624 \pm 477$ quilocalorias. Entre os pacientes operados por câncer, os submetidos a gastrectomia subtotal e Y-de-Roux apresentaram os maiores índices de massa corporal. Não houve relação do tempo decorrido desde a cirurgia com o índice de massa corporal ou com a ingestão calórica. Conclusões - Entre os pacientes operados por câncer, gastrectomia subtotal com reconstrução Y-de-Roux se associa com melhor estado nutricional. Outros fatores, que não a baixa ingestão de calorias, causam a perda de peso nos pacientes submetidos a gastrectomia.

DESCRITORES - Avaliação nutricional. Estado nutricional. Gastrectomia.

\section{INTRODUÇÃO}

A baixa taxa de sobrevivência de pacientes com câncer gástrico induziu cientistas a descrever o tipo de cirurgia requerida e a salientar a importância do diagnóstico precoce ${ }^{(8)}$. As diversas técnicas de reconstrução após gastrectomia não têm apenas finalidade de reconstruir a continuidade do trânsito digestivo, mas também a de compensar a perda de funções decorrente da retirada total ou parcial do estômago ${ }^{(11)}$. Os efeitos da gastrectomia total (GT) e subtotal (GST) sobre a qualidade de vida são freqüentemente ignorados ${ }^{(15,27)}$.

$\mathrm{O}$ aspecto nutricional é crucial na cirurgia de ressecção gástrica e o método mais adequado de reconstrução do canal alimentar para cada tipo de cirurgia deve ser considerado para se reduzir o risco de desnutrição pós-gastrectomia ${ }^{(21)}$. Os sinais e sintomas relacionados ao estado nutricional mais comumente observados nos pacientes com gastrectomia são anorexia, diarréia, síndrome de dumping, perda de peso, anemia e desnutrição protéico-energética ${ }^{(24)}$.

A perda de peso dos pacientes depende da extensão da ressecção gástrica, da preservação do piloro e do tipo de vagotomia realizada. Diminuição de ingestão de alimentos é a causa mais importante e pode ser secundária à saciedade precoce, dispepsia, perda de apetite, fatores socioeconômicos ou à restrição voluntária da ingestão pelo paciente para evitar sintomas $^{(6,24,27)}$. No entanto, alguns estudos demonstram que a perda acentuada de peso, de proteínas e de gorduras corporais dos pacientes ocorre principalmente já antes da cirurgia ${ }^{(6,14)} \mathrm{e}$ alguns autores afirmam que a desnutrição não é inevitável, contanto que se mantenha uma ingestão alimentar adequada $^{(7,10,13,20,30)}$. Contudo, a perda de peso em pacientes sem doença maligna com ingestão dietética adequada indica que outros fatores advindos da reconstrução pós-gastrectomia limitam a recuperação do peso corporal ${ }^{(6)}$.

Trabalho realizado no Hospital das Clínicas da Faculdade de Medicina de Ribeirão Preto, SP.

Endereço para correspondência: Nutr. Mayra Mayumi Kamiji - Rua Manoel Achê, 771/04 - Jardim Irajá - 14020-590 - Ribeirão Preto - SP. e-mail: maykamiji@hotmail.com 
A causa da desnutrição após gastrectomia não é bem determinada, mas sabe-se que os mecanismos que levam à desnutrição são multifatoriais ${ }^{(27)}$. Alguns dos fatores contribuintes são ingestão calórica inadequada, insuficiência pancreática exócrina, assincronismo entre alimentos e suco pancreático secretado, supercrescimento bacteriano, alterações na mucosa intestinal, tempo de trânsito intestinal reduzido e progressão da doença maligna em paciente gastrectomizados $^{(1,2,6,7,13,14,22,23)}$.

A insuficiência pancreatobiliar representa mecanismo de má assimilação dos nutrientes. Em caso de pancreatectomia, a má absorção de proteína e de gordura é um resultado constante. Em reconstruções em que se exclui a passagem de alimentos pelo duodeno, a liberação de bile e de enzimas pancreáticas e a sua mistura com alimentos ingeridos são retardadas, não ocorrendo adequada digestão ${ }^{(8)}$.

O crescimento bacteriano é outro mecanismo responsável por vários aspectos da má absorção. A desconjugação de sais biliares por bactérias anaeróbias previne a formação de micelas, dificultando o transporte de gorduras hidrolisadas através da mucosa intestinal, assim causando esteatorréia ${ }^{(9)}$. A má absorção de proteínas também pode resultar de crescimento bacteriano, presumivelmente por desaminação bacteriana e conversão de nitrogênio da proteína dietética em uréia ${ }^{(24)}$.

Há poucos estudos prospectivos sobre a qualidade de vida após gastrectomia e o impacto sobre a qualidade de vida em longo prazo causado por diferentes técnicas de reconstrução é ainda controverso ${ }^{(28)}$. A recurrência de carcinoma gástrico e as complicações no pós-operatório podem afetar a ocorrência de sintomas e o estado nutricional; é difícil de se estabelecerem possíveis vantagens ou desvantagens de qualquer reconstrução em estudo de curto seguimento dos pacientes ${ }^{(19)}$

O objetivo do presente trabalho foi comparar o impacto dos diferentes tipos de reconstrução do tubo digestivo após a gastrectomia sobre o estado nutricional.

\section{MÉTODOS}

\section{Pacientes}

Este estudo foi conduzido no Hospital das Clínicas da Faculdade de Medicina de Ribeirão Preto, SP, entre abril de 2001 a junho de 2002. Foram avaliados 50 pacientes atendidos nos ambulatórios de Gastroenterologia, submetidos a gastrectomia 0,5-39 anos antes, com mediana de 8,5 anos de pós-cirúrgico. Pacientes em quimioterapia pós-cirúrgica adjuvante e pacientes com suspeita ou confirmação de recurrência de câncer foram excluídos. Os dados do pré-cirúrgico foram obtidos por revisão dos prontuários. Os tipos de cirurgia e suas indicações estão distribuídos na Tabela 1.

\section{Avaliação nutricional e dietética}

Para as avaliações nutricional e dietética, foram utilizadas:

a) Avaliação nutricional subjetiva (ANS) $)^{(12)}$.

b) Avaliação antropométrica - peso, altura, índice de massa corporal (IMC), pregas cutâneas tricipital (PCT) e subescapular, circunferência muscular do braço (CMB) e área muscular do braço (AMB) ${ }^{(17)}$.

c) Avaliação dietética pelo método de recordatório alimentar.

d) Adequação da ingestão dos micronutrientes ferro e cálcio, a partir dos valores do Dietary Reference Intakes (DRI) ${ }^{(29)}$.

e) Quantificação dietética por meio do programa de avaliação nutricional e prescrição de dietas Diet $\mathrm{PRO}^{\circledR}$, versão 3.0.

\section{Análise estatística}

Utilizou-se o programa GraphPad InStat ${ }^{\circledR}$, versão 3.05. Os testes de Mann-Whitney, Kruskal-Wallis, Wilcoxon e o teste Spearman foram aplicados. Probabilidades menores que 0,05 foram aceitas como significantes.

TABELA 1 - Distribuição dos tipos de cirurgia conforme as indicações cirúrgicas

\begin{tabular}{lcccccc}
\hline & \multicolumn{3}{c}{ Gastrectomia subtotal } & \multicolumn{2}{c}{ Gastrectomia total } \\
\cline { 2 - 7 } Indicação & BII & BI & Henley & Y-de-Roux & BII & Y-de-Roux \\
\hline Câncer gástrico & 6 & & 5 & 1 & 1 \\
Úlcera gástrica & 13 & 5 & 2 & & \\
Neoplasia de papila & 1 & & & & 1 \\
Úlcera duodenal & 4 & 2 & 1 & 5 & 9 \\
Gastrinoma ZE & 1 & & & & \\
Total & 25 & 7 & 3 & & & \\
\hline
\end{tabular}

$\mathrm{ZE}=$ Zollinger-Ellison

$\mathrm{B}=$ Billroth 


\section{RESULTADOS}

Trinta e três $(66 \%)$ pacientes eram do sexo masculino. A idade variou de 20 a 82 anos, com mediana de 57 anos. As indicações cirúrgicas foram: 22 pacientes com câncer, 28 com úlcera gástrica ou duodenal, e 1 com gastrinoma Zollinger-Ellison. O IMC médio foi de $22 \pm 4,75 \mathrm{~kg} / \mathrm{m}^{2}$. Distribuição dos pacientes que apresentaram percentil de adequação $\leq 5$ para medidas de PCT, CMB e AMB encontram-se na Tabela 2. Nove (18\%) queixaram-se de sintomas gastrointestinais, sendo plenitude epigástrica o mais freqüente, seguido de náusea e perda de apetite. Não houve diferença significativa na freqüência de ocorrência de sintomas entre os tipos de reconstruções cirúrgicas. Apesar dos sintomas referidos, os pacientes que os apresentaram mantinham ingestão calórica semelhante àquela observada no conjunto de todos os pacientes.
A ingestão diária média foi de $1624 \pm 477 \mathrm{kcal}$ ou $28 \mathrm{kcal} / \mathrm{kg}$ de peso corporal, sendo que 21 (42\%) pacientes consumiam $31 \mathrm{kcal} / \mathrm{kg}$ de peso/ dia ou mais (Figura 1). A ingestão média de proteínas foi de $1,34 \mathrm{~g} / \mathrm{kg}$ de peso ou $18 \%$ do valor calórico consumido. As médias de adequação da ingestão de cálcio e de ferro foram 23,8\% e 151,3\% do DRI, respectivamente. A baixa ingestão de cálcio deveu-se a pacientes que não referiram consumo de leite e derivados no recordatório alimentar. $\mathrm{O}$ número de refeições foi de três a seis, com média de cinco por dia.

Considerando-se todos os pacientes, não houve diferença significativa entre as reconstruções cirúrgicas quanto aos resultados das avaliações nutricional e dietética. Houve correlação negativa entre ingestão calórica e IMC (Figura 2). Não se observou relação significativa entre o tempo pós-cirúrgico com o IMC ou com a ingestão calórica.

TABELA 2 - Distribuição dos pacientes que apresentaram percentil de adequação $\leq$ a 5 para prega cutânea tricipital, circunferência muscular do braço e área muscular do braço entre os tipos de reconstruções cirúrgicas

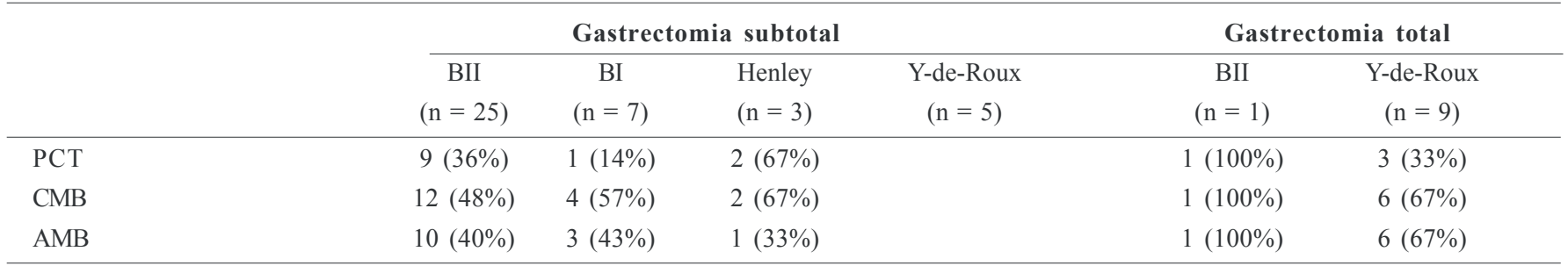

$\mathrm{PCT}=$ prega cutânea tricipital

$\mathrm{CMB}=$ circunferência muscular do braço

$\mathrm{AMB}=$ área muscular do braço $\quad \mathrm{B}=$ Billroth

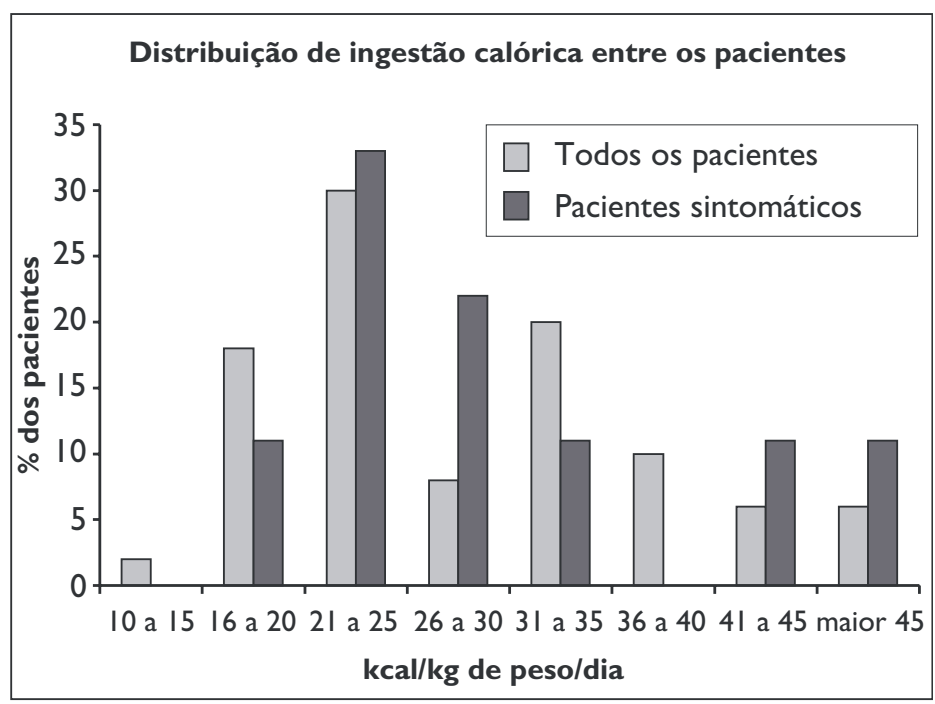

FIGURA 1 - Distribuição da ingestão calórica por quilo de peso corporal, entre os 50 pacientes

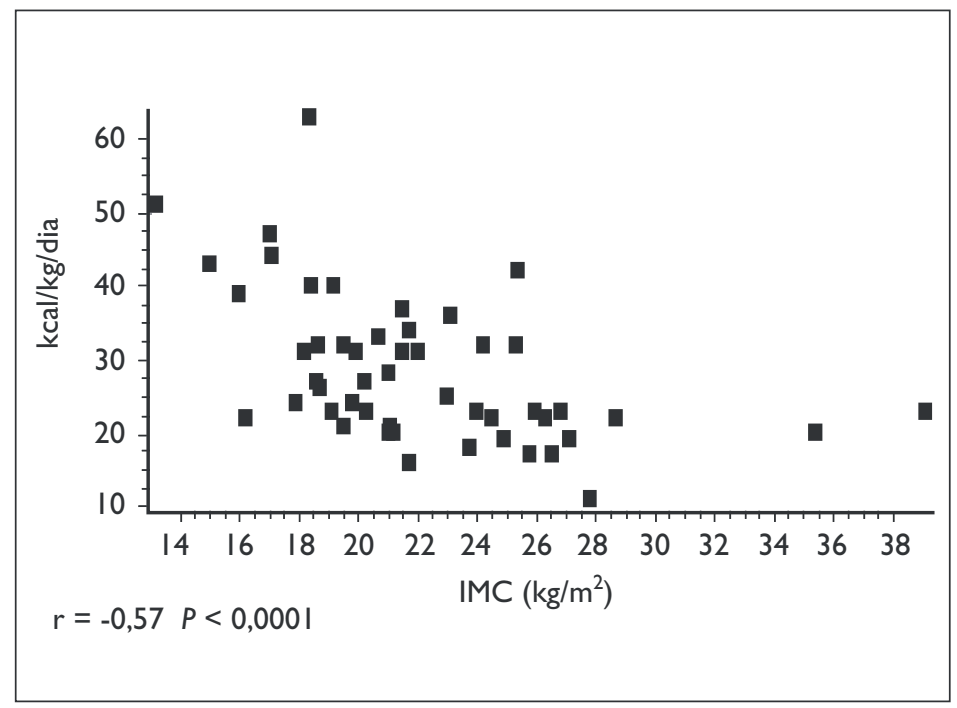

FIGURA 2 - Relação entre IMC e a ingestão calórica entre os pacientes 
Os valores de hemoglobina entre os pacientes submetidos as diferentes reconstruções cirúrgicas pós-GST foram (média \pm desviopadrão) $12,2 \pm 2 \mathrm{~g} \mathrm{~d} / \mathrm{L}$ naqueles com $\mathrm{BII}, 12,8 \pm 2,5 \mathrm{~g} / \mathrm{dL}$ naqueles com BI, 13,5 \pm 1 g/dL com reconstrução a Henley, $13 \pm 2$ g/dL nos pacientes com Y-de-Roux. Entre os gastrectomizados totais, o valor de hemoglobina foi $13 \pm 2 \mathrm{~g} / \mathrm{dL}$.

Segundo o resultado da ANS, seis (12\%) pacientes foram classificados como "potenciais desnutridos ou desnutridos moderados". Nestes, o percentil de adequação foi menor ou igual a 5 para $66,7 \%$ em PCT, e para todos em CMB. O IMC médio entre estes pacientes foi de $18,5 \mathrm{~kg} / \mathrm{m}^{2}$.

Não houve diferença significativa quanto aos dados das avaliações nutricional e dietética entre os pacientes operados por câncer e os operados por doença benigna, ou entre os pacientes submetidos a GT e a GST. Entre os pacientes submetidos a GST, aqueles com Y-deRoux apresentaram menor ingestão diária de calorias e maior IMC, este último já presente no pré-cirúrgico (Tabela 3).

\section{Pacientes operados por câncer}

Após a cirurgia, diferentemente do observado no pré-cirúrgico, aqueles com GT apresentaram menor IMC do que os com GST. Comparando-se os IMC no pré e no pós-cirúrgico, observa-se redução significativa de IMC nos pacientes com GT, o que não foi observado nos pacientes com GST (Tabela 4). Entre os pacientes com GST, observaram-se maiores IMC (Tabela 5) e CMB naqueles com Y-deRoux, comparativamente aos com Billroth II. Entre os pacientes operados por câncer com Y-de-Roux, aqueles com GST apresentaram maior IMC comparados aos com GT (Tabela 6). Não houve relação do tempo pós-cirúrgico com o IMC ou com a ingestão calórica e entre os pacientes submetidos a GT, não houve relação entre IMC e ingestão calórica.

Não se observaram diferenças significativas das avaliações nutricional e dietética entre os tipos de reconstruções cirúrgicas nos pacientes com diagnóstico de doença benigna.

TABELA 3 - Ingestão diária de calorias e de índice de massa corporal observados nos diferentes tipos de reconstrução cirúrgica nos pacientes submetidos a gastrectomia subtotal

\begin{tabular}{lcccc}
\hline & Billroth I & Billroth II & Henley & Y-de-Roux \\
\hline IMC pré-cirúrgico & $26,6 \pm 3,9$ & $22,1 \pm 3,9$ & $18,7 \pm 2,5$ & $30 \pm 8,8$ \\
IMC pós-cirúrgico & $22 \pm 4,1$ & $21,9 \pm 4,4$ & $18,4 \pm 3,2$ & $28 \pm 6$ \\
IDC & $24,7 \pm 7,9$ & $30 \pm 10,7$ & $33,7 \pm 8,3$ & $19,2 \pm 5,2$ \\
\hline
\end{tabular}

$\mathrm{IMC}=$ índice de massa corporal $\left(\mathrm{kg} / \mathrm{m}^{2}\right)$

IDC = ingestão diária de calorias, em quilocalorias/peso corporal/dia

TABELA 4 - Resultados de IMC no pré-cirúrgico e no pós-cirúrgico entre os pacientes operados por câncer

\begin{tabular}{ccc} 
& Gastrectomia subtotal & Gastrectomia total \\
\hline IMC no pré-cirúrgico & $25,7 \pm 7,3$ & $22,8 \pm 3,9$ \\
IMC no pós-cirúrgico & $23,3 \pm 6,1$ & $19,6 \pm 2,5^{*}$ \\
\hline
\end{tabular}

$\mathrm{IMC}=$ índice de massa corporal, em $\mathrm{kg} / \mathrm{m}^{2}$

*P $<0,05$ em relação ao pré-cirúrgico, teste de Wilcoxon, bicaudal

TABELA 5 - Resultados de IMC no pré-cirúrgico e no pós-cirúrgico dos pacientes operados por câncer com gastrectomia subtotal

\begin{tabular}{lcc}
\hline & Billroth II & Y-de-Roux \\
\hline IMC no pré-cirúrgico & $22,6 \pm 4,3^{*}$ & $30,2 \pm 8,8$ \\
IMC no pós-cirúrgico & $19,9 \pm 3,7$ & $28,6 \pm 6$ \\
\hline
\end{tabular}

*No grupo de pacientes com Billroth II, não foi encontrado registro do IMC pré-cirúrgico de um dos pacientes, portanto há menos um paciente comparativamente ao pós-cirúrgico 
TABELA 6 - Resultados de IMC no pré-operatório e no pós-operatório dos pacientes operados por câncer, com reconstrução a Y-de-Roux

\begin{tabular}{ccc}
\hline & GST & GT \\
\hline IMC no pré-cirúrgico & $30,2 \pm 8,8$ & $22,8 \pm 3,9$ \\
IMC no pós-cirúrgico & $28,6 \pm 6$ & $20 \pm 2,3^{*}$ \\
\hline
\end{tabular}

*P $<0,05$ em relação ao pré-cirúrgico, teste de Wilcoxon, bicaudal

\section{DISCUSSÃO}

Existem controvérsias em relação aos métodos mais adequados, sensíveis e específicos para se realizar a avaliação do estado nutricional $^{(18)}$. Cada um deles tem características próprias, que lhes conferem vantagens e/ou desvantagens sem que, contudo, haja o teste considerado padrão-ouro ${ }^{(12)}$. No presente estudo, entre os pacientes considerados desnutridos pela ANS, observaram-se valores antropométricos mais baixos comparativamente aos do grupo total. Entretanto, considerando somente os indicadores antropométricos, a prevalência de pacientes considerados desnutridos poderia ser maior, uma vez que maior número de pacientes apresentou valores de adequações para pregas cutâneas menores ou iguais a 5. Ao que parece, existe tendência de os resultados oferecidos pela ANS subestimarem aqueles encontrados pelos métodos antropométricos ${ }^{(12)}$. PAPINI-BERTO et al. ${ }^{(25)}$, em estudo recentemente publicado, utilizaram a combinação de vários indicadores nutricionais antropométricos e bioquímicos na avaliação nutricional dos pacientes com gastrectomia. Esses autores encontraram prevalência maior de desnutridos em comparação ao presente estudo e sugerem que a classificação nutricional seja feita pela associação de indicadores nutricionais, de fundamentação metodológica diferente, a fim de se superarem as limitações de cada indicador.

No estudo presente, observou-se correlação negativa entre IMC e ingestão calórica. Estudo envolvendo pacientes pós-GT revelou que alguns recuperaram peso corporal com ingestão alimentar inferior à metade da quantidade ingerida antes da cirurgia ${ }^{(20)}$. A maioria deles seguia o hábito alimentar familiar e não foi orientada quanto ao tipo e densidades calórica e protéica das preparações, à digestibilidade e fracionamento da dieta. Em conseqüência, dificilmente aumentariam suas reservas energéticas corporais, mesmo com ingestão calórica alta ${ }^{(20)}$.

Estudo prospectivo ${ }^{(8)}$ analisando 174 pacientes com GT revelou decréscimo de IMC médio desde o pré-operatório, apesar de ingestão calórica elevada. Conforme os autores, o maior consumo alimentar poderia ser meio subconsciente de recuperação pelos pacientes com perda acentuada de peso, o que poderia ter ocorrido neste estudo ${ }^{(8)}$. Outras possibilidades seriam a má digestão e má absorção dos alimentos ingeridos, e a má assimilação dos nutrientes. Estes poderiam ser resultantes de ausência ou restrição de reservatório gástrico, trânsito intestinal acelerado, supercrescimento bacteriano, insuficiência pancreática, conseqüentes ao procedimento cirúrgico ${ }^{(6,13)}$. Ou ainda, a dificuldade de pacientes em recuperar peso corporal, apesar de ingestão energética quantitativamente adequada, pode estar associada à progressão da doença maligna ${ }^{(6)}$.

A baixa ingestão de cálcio observada pode refletir hábito alimentar caracterizado por baixo consumo de leite e derivados, relacionado ou não à intolerância ao leite associada à gastrectomia ${ }^{(8)}$.

Entre os pacientes com GST, aqueles com Y-de-Roux apresentaram melhores parâmetros antropométricos, comparados aos com Billroth II. Estes dados sugerem que a reconstrução em Y-de-Roux promove melhor estado nutricional aos pacientes. Pacientes com reconstrução a Billroth II submetem-se ao déficit do potencial digestivo cloridropéptico, a destruição dos mecanismos regulatórios pilóricos, a dessincronização dos estímulos mecânicos, químicos e hormonais gastrointestinais $^{(16)}$, ao refluxo excessivo de conteúdo duodenal ou jejunal para o coto gástrico, envolvendo ou não sintomas de náusea, epigastralgia e vômitos biliosos. Entretanto, a presença de diferença já no período pré-cirúrgico entre os grupos correspondentes às diferentes reconstruções é compatível com a possibilidade de que o estado nutricional tenha influenciado a decisão do tipo de reconstrução cirúrgica a que o paciente seria submetido. Além disso, é possível que a perda de peso tenha ocorrido principalmente antes da cirurgia, mantendo-se posteriormente ${ }^{(6,8)}$

Não se observou diferença quanto aos resultados das avaliações nutricional e dietética entre os conjuntos de pacientes com GT e GST. Estes dados apontam no mesmo sentido de estudo que avaliou o estado nutricional no aspecto bioquímico e a ingestão dietética de pacientes gastrectomizados ${ }^{(26)}$. O estudo mostrou ingestão dietética semelhante entre os pacientes com GT e GST e concluiu não haver diferença quanto ao estado nutricional entre os mesmos. No estudo de PAPINIBERTO et al. ${ }^{(25)}$, assim como no presente, não foi observada diferença significativa de ingestão energética-protéica entre os pacientes submetidos a gastrectomia total e subtotal, e não houve relação entre tempo pós-cirúrgico e ingestão energética. Tal relação poderia ser conseqüente da adaptação fisiológica pelos pacientes à cirurgia ou, ainda, poderia estar presente no pós-operatório imediato, que não foi incluído no presente estudo.

Perda de peso é conseqüência regular da GT ou GST para câncer gástrico ${ }^{(28)}$. Entre os pacientes operados por câncer, aqueles com GST apresentaram melhores parâmetros antropométricos que aqueles com GT. A retirada total do estômago resulta em prejuízo na secreção 
cloridropéptica e do fator intrínseco, na função de reservatório alimentar, na função motora de trituração e homogeneização dos alimentos e no retardo do trânsito digestivo alto ${ }^{(11)}$. Pacientes com GT para câncer apresentam menor tolerância a alimentos comuns, mais sintomas pósprandiais, ingestão de refeições menores ${ }^{(4,30)}$ e recuperação de peso pós-operatório mais tardio ${ }^{(15)}$. Todos os pacientes com GT operados por câncer no presente estudo foram submetidos a Y-de-Roux, reconstrução mais realizada dada sua simplicidade, e não a ausência de distúrbios pós-prandiais ${ }^{(4)}$. Uma desvantagem da GST é a exposição dos pacientes ao risco de recurrência de câncer ${ }^{(3)}$; entretanto, ela está associada a melhor estado nutricional e qualidade de $\operatorname{vida}^{(5,15)}$.

No presente estudo, não se utilizaram instrumentos específicos que objetivam a detecção de sintomas nos pacientes, fato que, somado à exclusão de pacientes no período pós-operatório precoce, pode justificar a baixa prevalência de sintomas. Estudos que revelam prevalência mais alta de sintomas como diarréia, indigestão, disfagia e perda de apetite em pacientes com GT e Y-de-Roux utilizaram escalas que investigam, especificamente, sintomas gastrointestinais e sintomas associados à alimentação, que são mais freqüentes no pós-operatório precoce $^{(27,28)}$

Em conclusão, entre os pacientes operados por câncer, a GST e a reconstrução Y-de-Roux associam-se com melhor estado nutricional. Outros fatores, como má digestão dos alimentos ingeridos, má assimilação dos nutrientes, progressão da doença maligna e não à baixa ingestão de calorias são causas da perda de peso nos pacientes submetidos a gastrectomia.

Kamiji MM, Oliveira RB. Nutritional status and dietary assessment of patients with gastrectomy. Arq Gastroenterol 2003;40(2):85-91.

ABSTRACT - Background - Nutrition is a crucial factor in gastric resection surgery and the most suitable alimentary canal reconstruction method must be considered in order to reduce the risk of malnutrition. The cause of postgastrectomy malnutrition has not been clearly determined, but the mechanisms behind malnutrition are evidently multifactorial. Aim - To evaluate the nutritional status of patients who underwent different reconstructive procedures after total or subtotal gastrectomy. Patients and Methods - Fifty patients who have undergone gastrectomy for 0.539 years were assessed. The surgical procedures used were Billroth I in 7, Billroth II in 26, Henley in 3 and Roux-en-Y in 14 of the patients. Twenty one of them have followed gastrectomy for cancer. The nutritional status was evaluated by subjective global assessment, dietary recall and anthropometry. Results - According to subjective global assessment, 6 of 50 patients were mild malnourished. The mean body mass index was $22 \pm 4.75 \mathrm{~kg} / \mathrm{m}^{2}$, the average daily calorie intake was $1624 \pm 477 \mathrm{Kcal}$. Of the patients operated for cancer, those who underwent subtotal gastrectomy followed by Roux-en-Y presented higher body mass index. No relationship between the period of time since surgery with body mass index or with calorie intake was found. Conclusions - Among patients operated for cancer, subtotal gastrectomy with Roux-en-Y reconstruction is associated with better nutritional status. Factors other than low calorie intake are the cause of weight loss in patients with gastrectomy.

HEADINGS - Nutrition assessment. Nutritional status. Gastrectomy.

\section{REFERÊNCIAS BIBLIOGRÁFICAS}

1. Ambrecht U, Lundell L, Lindstendt G, Stockbrügger R. Causes of malabsorption after total gastrectomy with Roux-en-Y reconstruction. Acta Chir Scand 1988;154:37-41.

2. Basso N, Materia A, Gizzonio D. Nutritional effects of total gastrectomy. A prospective randomized study of Roux-en-Y vs. Longmire-Mouchet reconstruction. Ital J Surg Sci 1985;15:335-40.

3. Bozzetti F. Total versus subtotal gastrectomy in cancer of the distal stomach: facts and fantasy. Eur J Surg Oncol 1992;18:572-9.

4. Bozzetti F, Bonfati G, Castellani R, Maffioli L, Rubino A, Diazzi G, Cozzaglio L, Gennari L. Comparing reconstruction with Roux-en-Y to a pouch following total gastrectomy. J Am Coll Surg 1996;183:243-8.

5. Bozzetti F, Marubini E, Bonfanti G, Miceli R, Piano C, Gennari L. Subtotal versus total gastrectomy for gastric cancer: five-year survival rates in a multicenter randomized Italian trial. Italian Gastrointestinal Tumor Study Group. Ann Surg 1997;226:613-20

6. Bradley EL, Isaacs J, Hersch T, Davidson ED, Millikan W. Nutritional consequences of total gastrectomy. Ann Surg 1975;182:415-29.

7. Braga M, Foppa L, Zuliani W, Cristallo M, Di Carlo V. Malnutrition after total gastrectomy. Minerva Chir 1990;45:625-9.

8. Bragelmann R, Armbrecht U, Rosemeyer D, Schneider B, Zilly W, Stockbrügger RW. Nutrient malassimilation following total gastrectomy. Scand J Gastroenterol 1996; Suppl 218:26-33

9. Broido PW, Gorbach SL, Nyhus LM. Microflora of the gastrointestinal tract and the surgical malabsorption syndromes. Surg Gynecol Obstet 1972;135:449-60.
10. Calomino N, Malerba M, Palasciano G, Cappeli A, Oliva G, Salvestrini F, Tanzini G. Total gastrectomy and malnutrition. Minerva Chir 1998;53:883-7.

11. Ceneviva R, Alves ALT. Gastrectomia total. In: Silva Jr OC, Zucoloto S, Beer J A, editores. Modelos experimentais de pesquisa em cirurgia. São Paulo: Robe; 1998. p.349-56.

12. Correia MITD. Avaliação nutricional subjetiva. Rev Bras Clin 1998;13:68-73.

13. Cristallo M, Braga M, Agape D, Primignani M, Zuliani W, Vecchi M, Murone M, Sirone M, Di Carlo V, De Franchis R. Nutritional status, function of the small intestine and jejunal morphology after total gastrectomy for carcinoma of the stomach. Surg Gynecol Obstet 1986;163:225-30.

14. Curran FT, Hill GL. Failure of nutritional recovery after total gastrectomy. Br J Surg 1990;77:1015-7.

15. Davies J, Johnston D, Sue-Ling H, Young S, May J, Grifth J, Miller G, Martin I. Total or subtotal gastrectomy for gastric carcinoma? A study of quality of life. World J Surg 1998;22:1048-55.

16. Faintuch J. Desnutrição no paciente gastrectomizado. Arq Gastroenterol 2002;39:1-2.

17. Frisancho A. New norms of upper limb fat and muscle areas of assessment of nutritional status. Am J Clin Nutr 1981;34:2542

18. Hill GL, Windsor JA. Nutritional assessment in clinical practice. Nutrition 1995;11 Suppl 2:198S-201S

19. Iivonen MK, Mattla JJ, Nordback IH, Matikainen MJ. Long-term follow-up of patients with jejunal pouch reconstruction after total gastrectomy. A randomized prospective study. Scand J Gastroenterol 2000;35:679-85.

20. Ishihara K. Long-term quality of life in patients after total gastrectomy. Cancer Nursing 1999;22:220-7. 
21. Li Destri G, Trombatore G, La Greca G, Rinzivillo C, Rodolico M, Desiderio C, Orsina N, Di Cataldo A, Puleo S. Total gastrectomy: nutritional status after different reconstruction techniques. An experimental study. J Surg Oncol 1992;49:98-102.

22. Liedman B, Andersson H, Bosaeus I, Hugosson I, Lundell L. Changes in body composition after gastrectomy: results of a controlled, prospective clinical trial. World J Surg 1997;21:416-20.

23. Liedman B, Hugosson I, Lundell L. Treatment of devastating postgastrectomy symptoms: the potential role of jejunal pouch reconstruction. Dig Surg 2001;18:218-21.

24. Papini-Berto SJ, Burini RC. Causas da desnutrição pós-gastrectomia. Arq Gastroenterol 2001;38:272-5.

25. Papini-Berto SJ, Maio R, Módolo AK, Santos MDB, Dichi I, Burini RC. Desnutrição protéico-energética no pacientes gastrectomizado. Arq Gastroenterol 2002;39:3-10.

26. Stael von Holstein C, Ibrahimbegovic E, Walther B, Akesson B. Nutrient intake and biochemical markers of nutritional status during long-term follow-up after total and partial gastrectomy. Eur J Clin Nutr 1992;46:265-72.
27. Svedlund J, Sullivan M, Liedman B, Lundell L, Sjördin I. Quality of life after gastrectomy for gastric carcinoma: controlled study of reconstructive procedures. World J Surg 1997;21:422-33.

28. Svedlund J, Sullivan M, Liedman B, Lundell L. Long term consequences of gastrectomy for patients' quality of life: the impact of reconstructive techniques. Am J Gastroenterol 1999;94:438-45.

29. Trumbo P, Yates AA, Schlickers S, Poos M. Dietary reference intakes: vitamin A, vitamin K, arsenic, boron, chromium, copper, iodine, iron, manganese, molybdenum, nickel, silicon, vanadium, and zinc. J Am Diet Assoc 2001;101:295-301

30. Wu CW, Hsieh MC, Lo SS, Lui WY, P'eng FK. Quality of life of patients with gastric adenocarcinoma after curative gastrectomy. World J Surg 1997;21:777-82.

Recebido em 14/11/2002. Aprovado em 24/2/2003. 